\title{
EFFECTIVENESS OF A NEW COMPOUND, BENEMID, IN ELEVATING SERUM PENICILLIN CONCENTRATIONS
}

\author{
By JAMES M. BURNELL AND WILLIAM M. M. KIRBY \\ (From the Department of Medicine, University of Washington School of Medicine, and the King \\ County Hospital, Seattle, Washington)
}

(Submitted for publication February 14, 1951; accepted April 9, 1951)

The rapid renal clearance of penicillin has made it difficult to maintain blood levels adequate to combat resistant organisms such as enterococci and many staphylococci. Diodrast (1), p-amino-hippurate $(\mathrm{PAH})(2)$, benzoic acid (3), sodium benzoate $(3,4)$, and carinamide $(5)$, have been found to block renal tubular excretion of penicillin with enhancement of blood levels. Of these compounds, only. carinamide has had wiedspread clinical use $(6-8)$. Its value has been limited because the large doses required make it unwieldy and often poorly tolerated (9). This disadvantage appears to have been overcome by the recent discovery of a new compound, Benemid (10), ${ }^{1}$ which is apparently effective in low doses, and in preliminary studies has been found to have little toxicity (11). This report is based on measurements of penicillin blood levels in 74 patients treated with intramuscular penicillin $\mathrm{G}$ or its procaine salt.

\section{MATERIALS AND METHODS}

The subjects studied were adult patients in the King County Hospital who were receiving penicillin for a wide variety of infections. Patients with renal disease or congestive heart failure were excluded. After control penicillin levels were obtained, Benemid was administered in doses of $0.5 \mathrm{gm}$. every six hours for at least 12 to 24 hours prior to the measurement of the post-Benemid serum levels. Diet, salt, and fluid intake were not controlled. The concentrations of penicillin in the serum were de-

\footnotetext{
1 Sharp and Dohme's trade name for p-(di-n-propylsulfamyl)-benzoic acid.
}

termined by a tube dilution method, using killing of a Group A hemolytic streptococcus as the endpoint (12).

\section{CALCULATIONS OF DATA OBTAINED}

Two methods were used to compute the increase in the penicillin levels after Benemid. The first method consisted of determining the increase found in each individual patient, and from these figures the average individual fold increase was calculated. The second method consisted of averaging all of the pre- and post-Benemid penicillin levels before computing the fold increase.

Average of individual fold increases: For each patient the fold increase of the serum penicillin level after Benemid was determined by dividing the post-Benemid level by the pre-Benemid level. These individual fold increases were then averaged. In a few instances there was a decrease rather than an increase following Benemid, and these fold decreases were subtracted from the total of the increases before dividing by the total number of patients.

Fold increase of the average levels: Serum penicillin levels before and after Benemid were averaged separately. The fold increase of the average levels was then determined by dividing the post-Benemid average by the preBenemid average.

\section{RESULTS}

The first group (Table I) consisted of $19 \mathrm{pa}$ tients who were receiving 300,000 units of procaine penicillin once daily. Levels before and after Benemid were measured on one occasion in each patient at three, 12 and 24 hours following a penicillin injection. Of the 57 occasions on which concentrations were determined before and after Benemid, there was an increase in the penicillin

TABLE I

Effect of Benemid on serum penicillin levels of 19 patients receiving 300,000 units of procaine penicillin once daily

Hours after penicillin injection:

Average serum level (units/cc.) before Benemid

Range

Average serum level (units/cc.) after Benemid

Range

Fold increase of average serum levels

Average of individual fold increases

Range

$\begin{array}{ccc}3 \text { hrs. } & 12 \text { hrs. } & 24 \text { hrs. } \\ 3.14 & 1.07 & 0.23 \\ 0.5-10.0 & <0.04-5.0 & <0.04-5.0 \\ 6.34 & 2.14 & 0.66 \\ 0.5-20.0 & <0.04-7.0 & <0.04-2.0 \\ 1.99 & 2.00 & 2.86 \\ 2.03 & 4.37 & 2.82 \\ 5 \text { fold decrease to } & 1 \text { to } 10 \text { fold } & 4 \text { fold decrease to } \\ 10 \text { fold increase } & \text { increase } & 10 \text { fold increase }\end{array}$


level following Benemid 39 times (68.4\%). In the other 18 instances, the levels remained the same, or were lower following Benemid (eight times at three hours; five times each at 12 and 24 hours). The averages of the individual fold increases were $2.03,4.37$, and 2.82 at three, 12 and 24 hours respectively, and the fold increases of the average levels were $1.99,2.00$, and 2.86 .

When the group was divided into patients over the age of 60 and those under the age of 60 , those over 60 had uniformly higher levels before Benemid, and also greater and more consistent increases in the levels following Benemid.

The second group (Table II) consisted of 27 patients treated with 300,000 units of procaine penicillin every 12 hours. Levels were measured three and 12 hours following a penicillin injection. Of the 54 determinations which were made before and after Benemid, there was an increase of the penicillin level following Benemid on 50 occasions $(92.6 \%)$. Three levels at the three hour interval were the same before and after Benemid, and in one patient the level was lower following Benemid. The averages of the individual fold increases were 2.62 and 3.08 at three and 12 hours respectively, and the fold increases of the average levels were 2.11 and 2.67 .

It was again noted that patients over the age of 60 had higher levels before Benemid, and a greater increase in penicillin concentrations following Benemid.

The third group consisted of five patients treated with 600,000 units of procaine penicillin every

TABLE II

Effect of Benemid on serum penicillin levels of 27 patients treated with 300,000 units of procaine penicillin every 12 hours

\begin{tabular}{lcc}
\multicolumn{1}{c}{ Hours after penicillin injection: } & $3 \mathrm{hrs}$. & $12 \mathrm{hrs}$ \\
$\begin{array}{l}\text { Average serum level } \\
\text { (units/cc.) before Benemid }\end{array}$ & 2.62 & 0.84 \\
$\begin{array}{l}\text { Range } \\
\begin{array}{l}\text { Average serum level } \\
\text { (units/cc.) after Benemid }\end{array}\end{array}$ & $0.2-7.0$ & $0.04-2.0$ \\
$\begin{array}{l}\text { Range } \\
\begin{array}{l}\text { Fold increase of the } \\
\text { average serum levels }\end{array}\end{array}$ & $0.7-10.0$ & $0.2-10.0$ \\
$\begin{array}{l}\text { Average of individual } \\
\text { fold increase }\end{array}$ & 2.11 & 2.64 \\
Range & 2.62 & 3.08 \\
& $\begin{array}{l}2 \text { fold decrease to } \\
7 \text { fold increase }\end{array}$ & $\begin{array}{c}1 \text { to } 10 \text { fold } \\
\text { increase }\end{array}$
\end{tabular}

TABLE III

Effect of Benemid on serum penicillin levels of 19 patients treated with 100,000 units of crystalline penicillin $G$ every three hours

Average serum level

(units/cc.) before Benemid

Three hour serum

- penicillin level

Range

0.48

Average serum level

(units/cc.) after Benemid

$<0.04-4.0$

Range

Fold increase of the average serum levels

$0.07-10.0$

Average of individual fold increases

2.98

5.32

Range

1 to 10 fold increase

eight hours. In each instance the level after Benemid exceeded the control level. The average levels at the end of the eight hour period were increased by Benemid from 3.2 units/cc. to 10.4 units/cc. The fold increase of the average levels and the average of the individual fold increases were both approximately 3.2 .

The fourth group (Table III) consisted of 19 female patients (average age 29) from the Gynecology Service who were treated with 100,000 units of crystalline penicillin every three hours. Serum penicillin levels were measured three hours after one of the penicillin injections. The levels following Benemid were higher in 18 patients $(94.6 \%)$, and in the 19th, the level was the same before and after Benemid. Four patients (22\%) had measurable levels only following Benemid. The average level before Benemid was 0.48 units/ cc.; the average level following Benemid was 1.43 units/cc. In this series the average of the individual fold increases (5.32) was appreciably larger than the fold increase of the average levels (2.98). The significance of this difference will be mentioned in the discussion.

The fifth group (Table IV) consisted of four patients who received $1,000,000$ units of crystalline penicillin every two hours. Two had pneumococcic meningitis, one had staphylococcic meningitis, and in the fourth the diagnosis was non-paralytic poliomyelitis. Serum penicillin levels were measured two hours following an injection of penicillin. The average serum level was increased by Benemid from 21 units/cc. to 73 units/cc. In two of the patients spinal fluid levels were determined both be- 
fore and after Benemid, and showed increases of 10 to 40 fold.

\section{TOXICITY}

The toxicity of Benemid appears to be of low order. Most of the patients in this study received Benemid for only 48 to 72 hours, and in all instances the dosage was $2 \mathrm{gm}$. per day. However, five patients received Benemid for over two weeks. In none of the patients was there any evidence of systemic toxicity. Intolerance was limited to one patient who vomited the medication, and one patient who complained of dizziness. No abnormal urinary findings were detected during or after Benemid administration.

\section{DISCUSSION}

The foregoing data clearly indicate that the oral administration of a new compound, Benemid, causes a significant elevation of serum penicillin levels. In the 139 instances in which a comparison was made, penicillin levels were 3.39 times higher after Benemid. This is comparable to the elevation obtained with carinamide, and is accomplished with a much smaller dose. The lower dosage requirement is probably largely responsible for the greater tolerance of Benemid, and will undoubtedly lead to a much wider general acceptance.

It should be noted that penicillin concentrations

TABLE IV

Effect of Benemid on serum and cerebrospinal fuid levels of four patients treated with one million units of penicillin every two hours

\begin{tabular}{l|c|c|c|c|c|c}
\hline \hline & \multicolumn{2}{|c|}{$\begin{array}{c}\text { Before Benemid } \\
\text { penicillin levels } \\
\text { (units/cc.) }\end{array}$} & \multicolumn{2}{|c|}{$\begin{array}{c}\text { After Benemid } \\
\text { penicillin levels } \\
(\text { units/cc.) }\end{array}$} & \multicolumn{2}{|l}{ Fold Increase } \\
\cline { 2 - 6 } & Serum & CSF & Serum & CSF & Serum & CSF \\
\hline B. L. & 10 & & 50 & 7.0 & & \\
& 10 & & 50 & 7.0 & 5.7 & \\
C. U. & 7 & & 40 & & & \\
& 10 & 0.2 & 40 & 4.0 & & 20.0 \\
& & & 40 & 2.0 & 5.6 & 10.0 \\
J. O. & 40 & & & & & \\
& 20 & & 70 & 0.05 & 2.3 & \\
H. A. & 50 & 0.05 & 100 & 2.00 & 3.0 & 40.0 \\
& & & 200 & & & \\
\hline Average & 21 & 0.12 & 73 & 2.76 & 4.15 & 23.3 \\
\hline
\end{tabular}

were not invariably higher after Benemid administration. The least favorable results from this standpoint were obtained with procaine penicillin $(300,000$ units) injected once daily, in which an elevation occurred in only $68 \%$ of the instances. When procaine penicillin was administered twice daily, elevations occurred in $92 \%$, and with aqueous penicillin administered every three hours, enhancement was recorded in $95 \%$ of cases. The marked variability in absorption of penicillin, especially the procaine salt, undoubtedly accounts for these results, and it is not surprising that the greatest inconsistencies occurred when penicillin was injected only once daily.

In the present report, the enhancement of penicillin levels has been expressed mathematically in two ways, as the average of the individual fold increases, and as the fold increase of the average levels. Disparities between these two figures occurred in all major groups studied, and are a reflection of the marked variability of penicillin levels obtained in different patients. In any one patient, the results are usually fairly constant from day to day, but even under these circumstances there is enough variability in absorption to produce marked discrepancies. A good example occurred in Group 4, in which the average of the individual fold increases (5.32) was considerably higher than the fold increase of the average levels (2.98). Here, the exclusion of only one patient, whose pre- and post-Benemid penicillin levels were 4 and 10 units respectively, would have so altered the results that with both methods of calculation the final figure would have been approximately 5.3. The average of the individual fold increases would appear to be the most valid method of expressing the results, since it indicates the increment in levels in each patient. This point has seemed worthy of emphasis since it is not universally realized that there is a great variability in penicillin levels from patient to patient.

The administration of Benemid will probably contribute little to penicillin therapy in the majority of infections caused by penicillin sensitive organisms. However, there are certain conditions in which it may prove of great value in bringing about a favorable response, and in reducing the cost of treatment. Subacute bacterial endocarditis, especially when caused by penicillin resistant organisms such as enterococci, is one of the diseases 
in which a great deal may be accomplished by Benemid administration. Large amounts of penicillin are required to maintain levels as high as 20 units/cc. With Benemid, such levels may be elevated to 40 or 80 units/cc. without increasing the dosage of penicillin. In some cases this will undoubtedly bring about effective therapy, and will at the same time minimize the mechanical and economic problems of massive penicillin therapy. Infections with other penicillin resistant organisms, such as staphylococci and certain of the gram negative bacilli, also fall into this category. In the patients who received Benemid in addition to 1,000 ,000 units of crystalline penicillin every two hours, the blood levels were never lower than 40 units/cc. Concentrations of this magnitude should be effective in the therapy of certain infections which are not ordinarily considered susceptible to penicillin.

The results obtained in the four patients with meningitis were of special interest. Spinal fluid penicillin concentrations were elevated 10 to 40 fold in patients treated by the intramuscular route alone. The clinical response in these patients was striking, especially in a 43 year old woman who was seriously ill with staphylococcal meningitis. Benemid given in conjunction with large intramuscular doses of penicillin would appear highly efficacious in treating meningitis due to gram positive bacteria.

Finally, Benemid may find an important place in conjunction with oral penicillin therapy. The effectiveness of Benemid in prolonging therapeutic concentrations of penicillin should make it possible to achieve results with smaller doses, or with administration at greater intervals.

\section{SUMMARY AND CONCLUSIONS}

The effect of the oral administration of $0.5 \mathrm{gm}$. of Benemid every six hours on serum penicillin levels was measured in 74 patients. In 51 patients treated with procaine penicillin, there was enhancement of penicillin levels following Benemid in $81 \%$ of the instances, with an average fold increase of 2.87. In 23 patients treated with crystalline peni- cillin the levels were higher in $96 \%$ of the cases, with an average fold increase of 5.10. This three to five fold enhancement of penicillin levels should make the therapy of certain penicillin resistant infections considerably less expensive, and simpler from a mechanical standpoint.

\section{REFERENCES}

1. Rammelkamp, C. H., and Bradley, S. E., Excretion of penicillin in man. Proc. Soc. Exper. Biol. \& Med., 1943, 53, 30.

2. Beyer, K. H., Flippin, H. F., Verwey, W. F., and Woodward, R., Effect of para-aminohippuric acid on plasma concentration of penicillin in man. J.A.M.A., 1944, 126, 1007.

3. Bronfenbrenner, J., and Favour, C. B., Increasing and prolonging blood penicillin concentrations following intramuscular administration. Science, 1945, 101, 673.

4. Spaulding, E. H., Bondi, A., Jr., and Early, E., The effect of combining sodium benzoate with oral penicillins. Science, 1947, 105, 210.

5. Beyer, K. H., Miller, K. A., Russo, H. F., Patch, E. A., and Verwey, W. F., The inhibitory effect of carinamide on renal elimination of penicillin. Am. J. Physiol., 1947, 149, 355.

6. Boger, W. P., and Baker, R. M., Comparison of effect of carinamide and benzoic acid on penicillin plasma concentrations. Proc. Soc. Exper. Biol. \& Med., 1947, 66, 1.

7. Seeler, A. O., Wilcox, C., and Finland, M., Enhancement of blood levels by carinamide during intramuscular administration of penicillin. J. Lab. \& Clin. Med., 1947, 32, 807.

8. Strauss, E., Richburg, P. L., Saba, P. Z., and Alexander, J. E., Enhancement of plasma penicillin concentrations by carinamide and sodium benzoate. J. Lab. \& Clin. Med., 1947, 32, 818.

9. Boger, W. P., and Crosson, J. W., Toxicity of carinamide. Am. J. Med., 1950, 9, 35.

10. Beyer, K. H., Wiebelhous, V. D., Tillson, E. K., Russo, H. F., and Wilhoyte, K. M., 'Benemid', p(di-n-propylsulfamyl)-benzoic acid: inhibition of glycine conjugative reactions. Proc. Soc. Exper. Biol. \& Med., 1950, 74, 772.

11. Boger, W. P., Beatty, J. O., Pitts, F. W., and Flippin, H. F., The influence of a new benzoic acid derivative on the metabolism of para-aminosalicylic acid (PAS) and penicillin. Ann. Int. Med., 1950, 33, 18.

12. Kirby, W. M. M., and Rantz, L. A., Methods of measuring penicillin concentrations in body fluids. $\mathrm{J}$. Bact., 1944, 48, 603. 